\title{
Optimal experimental design on the loading frequency for a probabilistic fatigue model for plain and fibre-reinforced concrete
}

\author{
M. J. Rivas-López ${ }^{1}$, R. C. Yu ${ }^{2, *}$, J. López-Fidalgo ${ }^{3}$, G. Ruiz ${ }^{2}$ \\ ${ }^{1}$ Departamento de Estadística, Universidad de Salamanca \\ Plaza de los Caídos s/n, 37008 Salamanca, Spain \\ ${ }^{2}$ ETS de Ingenieros de Caminos, C. y P., Universidad de Castilla-La Mancha \\ Avenida Camilo José Cela s/n, 13071 Ciudad Real, Spain \\ ${ }^{3}$ Departamento de Matemáticas, Universidad de Castilla-La Mancha \\ Avenida Camilo José Cela s/n, 13071 Ciudad Real, Spain
}

\begin{abstract}
The objective is to improve the fatigue characterisation process based on the concept of optimal experimental design. This is carried out through a probabilistic model, previously developed, which takes into account the experimentally observed loading frequency effect on the fatigue life in plain and fibre-reinforced concrete. The Fisher Information Matrix is first obtained for the simplified fatigue model. The optimal design is found to be located at the minimum values allowed for both the maximum stress and stress ratio, whereas the two loading frequencies are the minimum and maximum values in the defined range. Next, the FIM is derived for the extended fatigue model. The previously carried out experimental plan is $65 \%$ efficient compared to the optimum. Even though it has been developed for the specific chosen fatigue model, the current procedure can be applied to any other fatigue model to significantly improve the fatigue characterisation process of any material.
\end{abstract}

Keywords: Fisher Information Matrix (FIM), D-optimality, Weibull cumulative distribution function, loading frequency, fatigue model

\footnotetext{
*Corresponding author: rena@uclm.es (E-mail), ETS de Ingenieros de Caminos, C. y P., Universidad de Castilla-La Mancha, Avenida Camilo José Cela s/n, 13071 Ciudad Real, Spain; Tel: +34 926295300 Ext. 6313.
} 


\section{Highlights}

- A general procedure is developed for obtaining the optimal design of a fatigue characterisation process.

- The Fisher Information Matrix is derived for both the simplified and complete fatigue models. The efficiencicy of the previously carried out experimental design is approximately $87 \%$ and $65 \%$ for the simplified and complete fatigue model, respectively.

- A robustness analysis is carried out for the obtained optimum. The efficiency surpasses $99 \%$ when the initial values fluctuated between $50 \%$ and $150 \%$ of the corresponding reference values. 


\section{Introduction}

Fatigue tests are known to be time consuming, and can sometimes be unachievable if not properly designed. The procedure on how to determine the number of tests needed to characterise certain materials is an open issue. In the current work, we make use of the Fisher Information Matrix (FIM) to derive the optimal location of tests to characterise fatigue performance of concrete-related materials under given loading conditions. In particular, the fatigue model based on an initial distribution developed by Saucedo et al. [1] is chosen as an example to carry out the optimal design process. The developed methodology, however, can be applied to any other given fatigue model.

Development of high-performance concrete for structures undergoing dynamic and cyclic loading has led to experiments conducted to study the influence of different fatigue parameters $[2,3,4,5,6,7,8,9,10,11,12,13$, $14,15,16,17,18,19]$. These parameters are related to either the fatigue test conditions, such as the minimum stress, $\sigma_{\min }$, the maximum stress, $\sigma_{\max }$, the loading frequency, $f$, or the material properties; for example the static material strength, $\sigma_{c}$, (compressive or tensile). In the case of concrete, it has been detected that the influence of the stress ratio, $R\left(\sigma_{\min }\right.$ divided by $\sigma_{\max }$ ), the loading frequency, $f$, and the stress level, $\sigma_{\max } / \sigma_{c}$, is quite relevant $[7,15,20,19]$.

Although the influence of loading frequency has been observed as early as the 1950s [3], the loading time [13] or frequency [14] was not included in the fatigue equation until the 1980s. Moreover, the static strength in concrete exhibits large dispersion and a Weibull distribution has been considered as the best distribution to fit fatigue life in concrete $[15,17]$. Recently Saucedo et al. [1] proposed a fatigue model to take into account the fact that, on the one hand, the static strength of concrete is a Weibull distribution; on the other hand, such a distribution can be considered as the limit behaviour when the fatigue life approaches one cycle. Meanwhile, influences of the loading frequency and of the stress ratio have also been incorporated. The application range of the proposed fatigue model is below $10 \mathrm{~Hz}$ according to experimental tests by Ruiz et al. [21, 19]. Depending on the effect of the loading frequency on the hardening exponent of the dynamic material strength is ignored or not, correspondingly the fatigue model is viewed as simplified or complete. We aim to explore the procedure to calibrate the model parameters based on the concept of optimal experimental design herein. 
Optimal experimental designs are especially useful when experimentation is expensive, time consuming or difficult to carry out. A good design will definitely save time, money and provide a better fitting of the model. An experimental design means selecting experimental conditions in such a way that they result in precise estimates or good predictions with a minimum of sample size, which in other words means adequately choosing the levels of the covariates. The optimality of a design is model-oriented in the sense that a design could be quite good for a particular model and not so good for a rival model. In the case of the existence of rival models a discrimination test may be performed to detect the most appropriate one. Optimal designs can be computed for discrimination purposes [31]. In reliability and survival analysis, the Cox proportional hazards model [22] is quite traditional, although proportionality is not always justified. The model is frequently fitted using partial likelihood. Optimal experimental designs in this context were recently computed by López-Fidalgo and Rivas-López [23]. Even though accelerated failure testing models have been increasingly used in recent years, experimental designs have not been studied as much in this field; see Rivas-López et al. [24]. In the current work, optimal experimental design is studied for the aforementioned fatigue model for concrete-like materials; in particular, to best estimate the model parameters under given loading conditions.

The rest of this paper is organised as follows. The concept of optimal experimental design is presented next. Applications to the simplified and complete fatigue model are developed in Section 3 and Section 4, respectively. Relevant conclusions are drawn in Section 5.

\section{Optimal experimental design}

Let $x$ be the vector of covariates, say an experimental condition (loading frequency, maximum stress and stress ratio for a fatigue test), which can be chosen from a compact design space, $\chi$; typically a product of intervals. For a value of $x$, a response variable time-to-event, $t$, (equivalently, the fatigue life, $N$ ), is observed. This is considered as a random variable from a parametric family of distributions, indexed by $\beta$, a vector of parameters. An exact design of size $n$ is defined by a collection of experimental conditions $x_{1}, x_{2}, \ldots, x_{n}$ in $\chi$, where some of these may be repeated. Thus, a probability measure can be defined with support on the distinct points of the design with weights proportional to the number of repetitions (replicates). This leads to an extension of this definition to any probability measure, $\xi$, the so called 
approximate design [26], which will be used in this work. An exact design of a particular size, say $n$, is what one can only put in practice using just $n$ experiments. Extending the concept to any probability measure (approximate design) means a kind of abstraction of the real world. This is made because the concept allows a mathematical result, which proves very useful to compute optimal designs. As a matter of fact, computing optimal exact designs is rather difficult and frequently convergence of the typical algorithms can not be proved. The main drawback is that once the optimal approximate design is computed, an exact design has to be obtained using some rounding procedure. For instance, for a discrete measure, $\xi$, the experimenter has to perform "approximately" $n_{i} \approx n \xi\left(x_{i}\right)$ experiments at $x_{i}$, in such a way $\sum_{i} n_{i}=n$. Imhof et al. [30] provided some examples to show that if the sample size is large, any rounding procedure leads to a quite efficient exact design. Otherwise, if $n$ is small, then the impact of the rounding may be quite important. Hereafter $n$ will be the total number of experiments to be performed, while $k$ will be the distinct experimental conditions, some of them replicated. An approximate design with $k$ different points in its support will be frequently called a $k$-point design.

The Fisher information is a way of measuring the amount of information that an observable random variable $x$ contains about an unknown parameter upon which the probability model of $x$ depends. The Fisher Information Matrix (FIM) associated with a finite design, $\xi(x)$, is

$$
M(\beta ; \xi)=\sum_{x} I(\beta ; x) \xi(x), \quad I(\beta ; x)=E\left(-\frac{\partial^{2} L L(\beta ; t, x)}{\partial \beta \partial \beta^{\prime}}\right),
$$

where $L L(\beta ; t, x)$ is the log-likelihood function of the observation $t$ for a particular experiment $x, \mathrm{I}(\beta ; x)$ is the corresponding Fisher Information Matrix, which is the expected value of the negative second derivative of $L L(\beta ; t, x)$. In particular, it is well known that restricting to approximate designs with finite support still preserves the whole space of information matrices. This is a consequence of the application of Carathéodory's theorem. Thus, we may limit ourselves to this type of design.

Since the covariance matrix (or dispersion matrix) of the estimators is asymptotically proportional to the inverse of the FIM [25], an optimality criterion will be based on a function of (the inverse of) the FIM. The most popular is D-optimality, which maximises the $m^{\text {th }}$-root of the determinant of the FIM, where $m$ is the number of parameters. Therefore, the D-optimal 
design is the design maximising the determinant of the FIM. The statistical interpretation of this criterion is that it minimises the volume of the confidence ellipsoid of the parameters of the model. It is easy to check that if the support of the D-optimal design is the number of parameters of the model, which is rather frequent, the determinant is the product of the weights of the design and of a function depending just on the support points. Then the product of the weights is maximised by equal weights. Thus, the best of this type of designs may be computed in an easy way. Consequently the so called General Equivalence Theorem (GET) [26] can be used to check whether this is actually the D-optimal design or not. The GET for D-optimality states that a design $\xi_{D}$ is D-optimal if and only if

$$
\psi\left(\xi_{D}, \beta ; x\right)=\operatorname{tr}\left[M^{-1}\left(\beta ; \xi_{D}\right) I(\beta ; x)\right] \leq m, x \in \chi .
$$

Moreover, equality is reached at the support points of the design.

If the model is non-linear then the FIM will depend on the parameters. Using nominal values of the parameters leads to the so called locally optimal designs. The way of using the GET to check D-optimality of a particular design $\xi$ means computing the FIM for this design, $M(\beta ; \xi)$, which, with specific nominal values of the parameters, will be a matrix with numerical entries. Then $I(\beta ; x)$ will be a matrix depending just on $x$ when the nominal values of the parameters are used. Thus, for a specific design and nominal values of the parameters, $\psi(\xi, \beta ; x)$ is just a function of $x$. It may be checked that this function is non-negative on the design space $x \in \chi$, either analytically or numerically, e.g. plotting it, if $\chi$ is one or two-dimensional.

A detailed introduction to the theory of optimal experimental design is provided, e.g., by Pázman [27], among others. Next, we search such designs for both the simplified and complete fatigue models.

\section{Applications to the simplified fatigue model}

In this Section, we first summarise the fatigue model developed by Saucedo et al. [1] for completeness. Then the concept of optimal experimental design is applied for the case when the effect of loading frequency on the hardening exponent of the dynamic material strength is ignored.

On the one hand, the distribution of the static compressive strength, $\sigma_{f_{0}}$, for a quasi-brittle material like concrete is shown to be a three-parameter 
140

Weibull distribution [1]

$$
F\left(\sigma_{f_{0}}\right)=1-\exp \left\{-\left(\frac{\sigma_{f_{0}}-\sigma_{m_{i n}}}{\lambda}\right)^{K}\right\},
$$

where the parameters $\sigma_{\text {min }_{0}}$ (location), $\lambda$ (scale) and $K$ (shape) are properties of the material and have to be estimated through static compressive tests. On the other hand, three limit conditions must be fulfilled for a fatigue test,

$$
\begin{aligned}
\lim _{N \rightarrow \infty} \sigma_{f} & =\sigma_{\min _{0}}, \\
\lim _{R \rightarrow 1} \sigma_{f} & =\sigma_{f_{0}}, \text { and } \\
\lim _{N \rightarrow 1} \sigma_{f} & =\sigma_{f_{0}} .
\end{aligned}
$$

The first limit condition reflects the fact that when the stress level reaches the fatigue endurance limit, $\sigma_{m_{i n}}$, an infinite number of cycles can be resisted without causing any damage to the material. The second limit condition states that, if the stress ratio, $R$, approaches one, the loading condition is actually static. Consequently, the material fails at its static strength, $\sigma_{f_{0}}$. In the same way, the third limit condition emphasises that, if the fatigue life $N$ is equal to one, the employed maximum stress level is necessarily the material's static strength, $\sigma_{f_{0}}$. If any of these three conditions is not satisfied for a fatigue model, the implication is that that model will not reflect physical reality at asymptotic conditions.

The following expression was proposed to relate the equivalent static strength of the fatigue load with the number of cycles resisted, $N$,

$$
\sigma_{f_{0}}=\sigma_{\text {min }_{0}}+\left(\sigma_{\max _{0}}-\sigma_{\text {min }_{0}}\right) N^{-[b+c \ln (1+f)](1-R)},
$$

where the effect of the loading frequency, $f$, has been taken into consideration.

According to the FIB Code [28], the dynamic stress $\sigma_{\max }$ can be related to its static counterpart by the equation,

$$
\sigma_{\max _{0}}=\sigma_{\max }\left(\frac{\dot{\sigma}_{0}}{2 f \Delta \sigma}\right)^{0.014} \equiv \sigma_{\max } d^{0.014},
$$

where 0.014 is the dynamic hardening coefficient, $\dot{\sigma}_{0}$ is the stress rate at which static characterisation tests were carried out, whereas

$$
d=\frac{\dot{\sigma}_{0}}{2 f \Delta \sigma}=\frac{\dot{\sigma}_{0}}{2 f(1-R) \sigma_{\max }} .
$$


then

$$
\begin{aligned}
\frac{\partial^{2} L L}{\partial b^{2}} & =-\frac{(1-R)^{2}}{B^{2}}-A^{K} N^{B K} K^{2}(1-R)^{2}[\ln (N)]^{2}, \\
\frac{\partial^{2} L L}{\partial b \partial c} & =\frac{\partial^{2} L L}{\partial b^{2}} \ln (1+f), \\
\frac{\partial^{2} L L}{\partial c^{2}} & =\frac{\partial^{2} L L}{\partial b^{2}}[\ln (1+f)]^{2},
\end{aligned}
$$

the cumulative distribution function of $N$ can be rewritten as

$$
F(N)=1-\exp \left\{-A^{K} N^{B K}\right\} .
$$

Thus, once the parameters of the Weibull distribution in Eq. (3) are estimated, the distribution of $N$ for a given test condition (where $\sigma_{\max }, R$ and $f$ are specified), depends on the two parameters $b$ and $c$, which are to be estimated.

The likelihood and log-likelihood functions for specific values of the parameter set, $\left(f, \sigma_{\max }, R\right)$, and fatigue life, $N$, are respectively

$$
\begin{aligned}
L(b, c) & =\frac{\partial F(N)}{\partial N}=A^{K} B K N^{B K-1} \exp \left\{-A^{K} N^{B K}\right\}, \\
L L(b, c) & =K \ln (A)+\ln (B)+\ln (K)+(B K-1) \ln (N)-A^{K} N^{B K} .
\end{aligned}
$$

$$
\begin{aligned}
& \frac{\partial B}{\partial b}=(1-R), \\
& \frac{\partial B}{\partial c}=(1-R) \ln (1+f)=\frac{\partial B}{\partial b} \ln (1+f),
\end{aligned}
$$


171

and the first element of the FIM, $I\left(b, c ; f, \sigma_{\max }, R\right)$, is

$$
\begin{aligned}
I_{11}\left(b, c ; f, \sigma_{\max }, R\right) & =E\left[-\frac{\partial^{2} L L}{\partial b^{2}}\right]=\int_{0}^{\infty}\left(-\frac{\partial^{2} L L}{\partial b^{2}}\right) L(N) d N \\
& =\frac{\left(\pi^{2} / 6\right)+\left[1-C_{E M}-K \ln (A)\right]^{2}}{[b+c \ln (1+f)]^{2}},
\end{aligned}
$$

where $E$ stands for the mathematical expectation according to the probability distribution of the data; that is, the integral of the quantity multiplied by the density function (or the likelihood function); and

$$
C_{E M}=\lim _{m \rightarrow \infty} \sum_{i=1}^{m}\left(\frac{1}{i}-\ln (m)\right) \simeq 0.577216
$$

represents the Euler-Masheroni constant [29]. For simplicity, we omit the arguments of the $i j$-th component of the FIM, $I\left(b, c ; f, \sigma_{\max }, R\right)$, by denoting it as $I_{i j}$. In addition, by defining $X(f)=\log (1+f)$, the remaining components are written as follows

$$
I_{12}=I_{21}=I_{11} X(f), \quad I_{22}=I_{11}[X(f)]^{2} .
$$

Therefore the FIM

$$
I\left(b, c ; f, \sigma_{\max }, R\right)=\phi \cdot \phi^{T}
$$

where

$$
\phi=I_{11}\left(\begin{array}{c}
1 \\
X(f)
\end{array}\right)
$$

which is the FIM of a linear model with these regressors. If the D-optimal design has two points, then their weights must be equal, as stated in the Introduction. We will consider a 2-point approximate design of this type,

$$
\xi=\left\{\begin{array}{cc}
\left(f_{1}, \sigma_{\max 1}, R_{1}\right) & \left(f_{2}, \sigma_{\max 2}, R_{2}\right) \\
1 / 2 & 1 / 2
\end{array}\right\} .
$$

The FIM is

$$
M(b, c ; \xi)=\frac{1}{2}\left(\begin{array}{ll}
\sum_{i=1}^{2} I_{11}\left(f_{i}, \sigma_{\text {maxi }}, R_{i}\right) & \sum_{i=1}^{2} I_{11}\left(f_{i}, \sigma_{\text {maxi }}, R_{i}\right) X\left(f_{i}\right) \\
\sum_{i=1}^{2} I_{11}\left(f_{i}, \sigma_{\text {maxi }}, R_{i}\right) X\left(f_{i}\right) & \sum_{i=1}^{2} I_{11}\left(f_{i}, \sigma_{\text {maxi }}, R_{i}\right) X\left(f_{i}\right)^{2}
\end{array}\right)
$$


Table 1: Model parameters for material C2 (plain concrete), CF1 (steel-fibre reinforced concrete) and CF2 (polypropylene-fibre reinforced concrete) and fatigue test conditions in [1] and [19].

\begin{tabular}{lcccccccc}
\hline Mat. & $b$ & $c$ & $\begin{array}{c}\lambda \\
{[\mathrm{MPa}]}\end{array}$ & $K$ & $\begin{array}{c}\sigma_{\min 0} \\
{[\mathrm{MPa} / \mathrm{s}]}\end{array}$ & $\begin{array}{c}\dot{\sigma}_{0} \\
{[\mathrm{MPa}]}\end{array}$ & $\begin{array}{c}\sigma_{\max } \\
{[\mathrm{MPa}]}\end{array}$ & $\begin{array}{c}R \\
-\end{array}$ \\
\hline C2 & 0.063 & -0.011 & 76.1 & 19.8 & 3.1 & 0.2 & 66.4 & 0.3 \\
CF1 & 0.049 & 0.0066 & 76.1 & 31.0 & 12.0 & 0.2 & 75.6 & 0.3 \\
CF2 & 0.052 & -0.001 & 70.2 & 15.8 & 4.8 & 0.2 & 62.9 & 0.3 \\
\hline
\end{tabular}

187

188

and its determinant

$$
\operatorname{det}(M)=\frac{1}{4} I_{11}\left(f_{1}, \sigma_{\max 1}, R_{1}\right) I_{11}\left(f_{2}, \sigma_{\max 2}, R_{2}\right)\left[X\left(f_{1}\right)-X\left(f_{2}\right)\right]^{2} .
$$

Since $I_{11}$ is non-increasing with respect to both $\sigma_{\max }$ and $R$, the best of these designs will be supported at the smallest possible values of them. It remains for the function to be maximised solely for the variable $f$.

For demonstration purposes, we apply the optimal design procedure to the plain concrete $\mathrm{C} 2$ and the reinforced concrete $\mathrm{CF} 1$ and $\mathrm{CF} 2$, referred in $[1,19]$ and shown in Table 1 . First we consider the design spaces $f \in$ $(0,10], \sigma_{\max } \in[0.5 \lambda, \lambda]$ and $R \in(0.0001,1]$, where 0.0001 is a small value chosen to avoid a zero stress ratio.

By maximising Eq. (10), the determinant of the FIM for each concrete, the best two-point designs with equal weights are obtained as follows

$$
\xi_{C 2}^{*}=\left\{\begin{array}{cc}
(0.0759,38.05,0.0001) & (10,38.05,0.0001) \\
1 / 2 & 1 / 2
\end{array}\right\}
$$

$$
\xi_{C F 1}^{*}=\left\{\begin{array}{cc}
(0.0339,38.05,0.0001) & (10,38.05,0.0001) \\
1 / 2 & 1 / 2
\end{array}\right\}
$$

$$
\xi_{C F 2}^{*}=\left\{\begin{array}{cc}
(0.0458,35.10,0.0001) & (10,35.10,0.0001) \\
1 / 2 & 1 / 2
\end{array}\right\} .
$$

The sensitivity function Eq. (2) is used to check whether these designs are actually D-optimal or not. In Fig. 1, we plot the sensitivity function and 


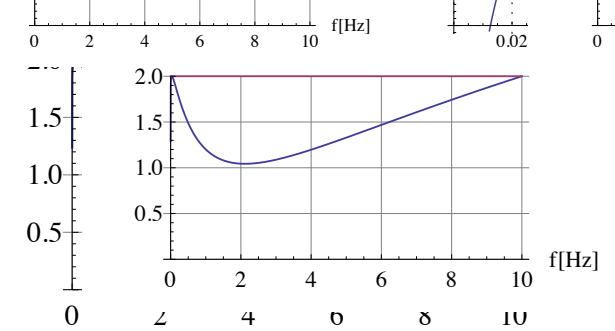

(a)

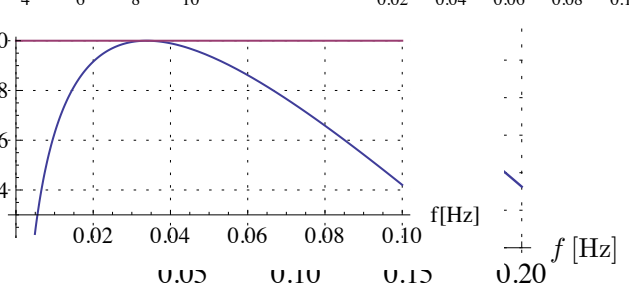

Sensitivity

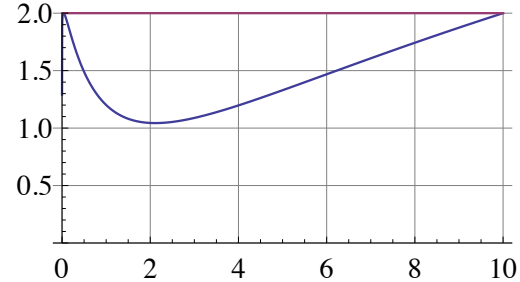

Sensitivity

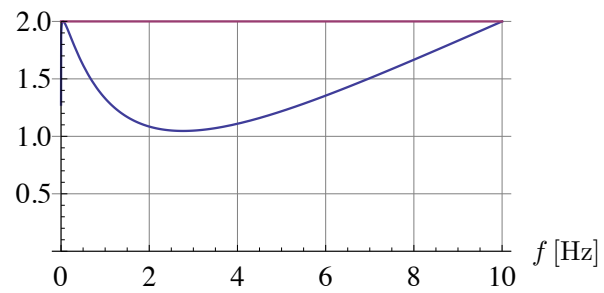

(b)

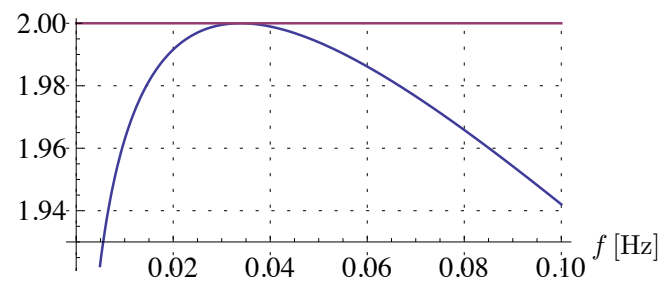

(c)

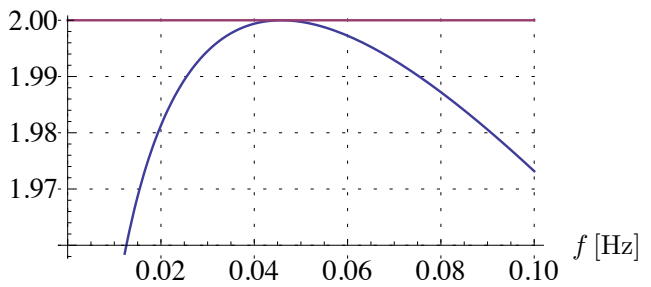

Figure 1: (For the simplified fatigue model) Sensitivity function for the D-optimal design over the entire frequency range, from 0 to $10 \mathrm{~Hz}$, (left column) and the zoomed view at the top-left corner (right column) for (a) $\mathrm{C} 2$, (b) CF1 and (c) CF2, where the maximum is located at $0.0759 \mathrm{~Hz}, 0.0339 \mathrm{~Hz}$ and $0.0458 \mathrm{~Hz}$, respectively.

the zoomed view of the top-left corner for $\mathrm{C} 2, \mathrm{CF} 1$ and $\mathrm{CF} 2$ respectively. As mentioned before, once the values of $\sigma_{\max }$ and $R$ were fixed, the maximum (two for a two-parameter design) is attained at two points of the design; one corresponds to a low-loading frequency, the other, the maximum frequency $(10 \mathrm{~Hz})$ allowed.

In order to check dependency on the choice of the nominal parameters, a robustness analysis is carried out. Suppose that the values of the real parameters are $\left(b_{t}, c_{t}\right)$. The FIM of the corresponding optimal design, $\xi_{t}^{*}$, for them is computed. Consider another design, for example one of those given in Eqs. (11-13), which we denote as $\xi_{n}$. The efficiency of the design $\xi_{n}$ is 
measured by

$$
E f f_{\left(b_{t}, c_{t}\right)}\left(\xi^{*}\right)=\left[\frac{\operatorname{det}\left(M\left(b_{t}, c_{t} ; \xi_{n}\right)\right)}{\operatorname{det}\left(M\left(b_{t}, c_{t} ; \xi_{t}^{*}\right)\right)}\right]^{\frac{1}{2}} .
$$

Sensitivity

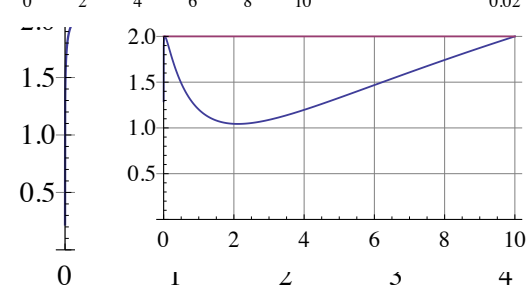

Sensitivity

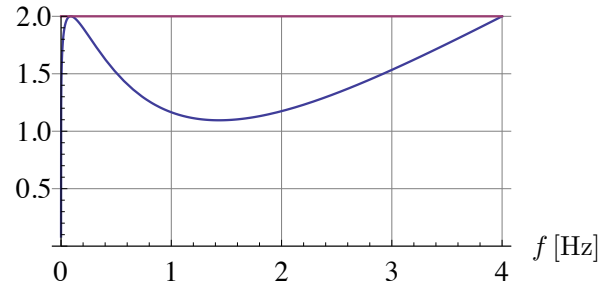

Sensitivity

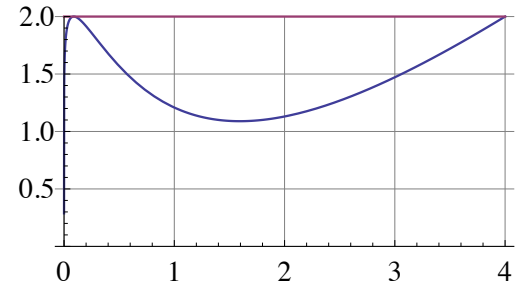

(a)

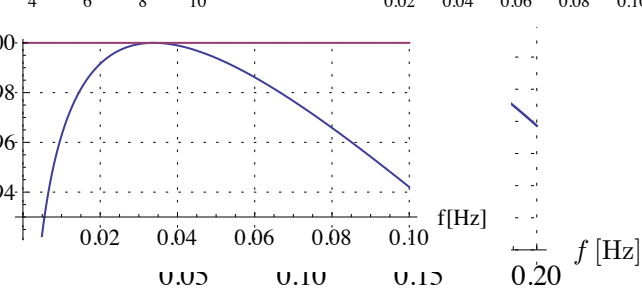

(b)

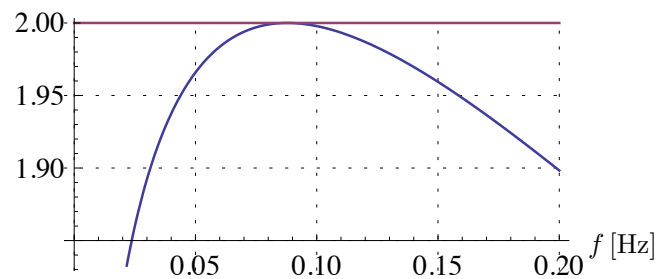

(c)

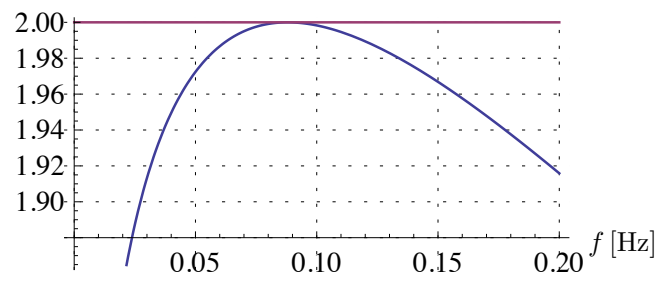

Figure 2: (Simplified fatigue model) Sensitivity function for the D-optimal design over the entire frequency range, from 0 to $4 \mathrm{~Hz}$, (left column) and the zoomed view at the top-left corner (right column) for (a) C2, (b) CF1 and (c) CF2, where the maximum is located at $0.1199 \mathrm{~Hz}, 0.0879 \mathrm{~Hz}$ and $0.0880 \mathrm{~Hz}$, respectively.

Since, in the experimental design, both the maximum stress, $\sigma_{\max }$, and the stress ratio, $R$, are fixed, as can be seen from Table 1 , we apply these values to carry out the optimisation procedure again. The following designs 
202

203

$$
\xi_{C F 2}^{*}=\left\{\begin{array}{cc}
(0.0880,62.9,0.3) & (4,62.9,0.3) \\
1 / 2 & 1 / 2
\end{array}\right\}
$$

$$
\xi_{C F 1}^{*}=\left\{\begin{array}{cc}
(0.0879,75.6,0.3) & (4,75.6,0.3) \\
1 / 2 & 1 / 2
\end{array}\right\}
$$

(see Fig. 2) are obtained:

$$
\xi_{C 2}^{*}=\left\{\begin{array}{cc}
(0.1199,66.4,0.3) & (4,66.4,0.3) \\
1 / 2 & 1 / 2
\end{array}\right\}
$$

204 205

205 for each material, that is,

$$
\xi=\left\{\begin{array}{cccc}
\left(4^{-2}, \sigma_{\max }, 0.3\right) & \left(4^{-1}, \sigma_{\max }, 0.3\right) & \left(4^{0}, \sigma_{\max }, 0.3\right) & \left(4^{1}, \sigma_{\max }, 0.3\right) \\
1 / 4 & 1 / 4 & 1 / 4 & 1 / 4
\end{array}\right\}
$$

where $\sigma_{\max }$ is a constant for each material. The efficiencies of this experimental design, relative to each of the above, are of $87.58 \%, 86.75 \%$ and $86.96 \%$ for $\mathrm{C} 2, \mathrm{CF} 1$ and $\mathrm{CF} 2$, respectively.

\section{Applications to the complete fatigue model}

Taking into account the loading frequency, Saucedo et al. [1] proposed a new relationship between dynamic and static compressive strengths; namely

$$
\sigma_{\max _{0}}=\sigma_{\max }\left(\frac{\dot{\sigma}_{0}}{2 f \Delta \sigma}\right)^{0.014 \exp \{\gamma f\}} \equiv \sigma_{\max } d^{0.014 \exp \{\gamma f\}},
$$

where the exponent was fitted for frequencies below $10 \mathrm{~Hz}$. The difference between the above equation and Eq. (5) lies in the fact that the hardening coefficient is explicitly dependent on the loading frequency. As a result, a new model parameter, $\gamma$, is added. Plugging Eq. (17) into Eq. (3) to determine the cumulative distribution function, which we term as the complete fatigue model, we obtain

$$
F(N)=1-\exp \left\{-\bar{A}^{K} N^{B K}\right\}
$$


where

$$
\begin{aligned}
& \bar{A}=S_{m x} d^{0.014 \exp \{\gamma f\}}-S_{m 0} \\
& B=[b+c \ln (1+f)](1-R)
\end{aligned}
$$

and $S_{m x}, S_{m 0}$ and $d$ are defined as in Section 3. Thus, once the parameters of the Weibull distribution are estimated, the distribution of $N$ depends on the variable $\sigma_{\max }$ and $R$ in the fatigue test and the frequency, $f$, as well as the parameters $b, c$ and $\gamma$.

In the previous section it was shown that the minimum values of $\sigma_{\max }$ and $R$ had to be included in the support of the $\mathrm{D}$-optimal design. For this reason, we expect the same will happen here again. So we will concentrate on the design for the loading frequency, $f$.

The log-likelihood function for a specific value of $f$ is

$$
L L(N)=K \ln (\bar{A})+\ln (B)+\ln (K)+(B K-1) \ln (N)-\bar{A}^{K} N^{B K},
$$

Since

$$
\begin{aligned}
\frac{\partial \bar{A}}{\partial \gamma} & =S_{m x} d^{0.014 \exp \{\gamma f\}} 0.014 \exp \{\gamma f\} f \ln (d) \\
& =0.014 \exp \{\gamma f\} f \ln (d)\left(\bar{A}+S_{m 0}\right), \\
\frac{\partial B}{\partial b} & =(1-R), \\
\frac{\partial B}{\partial c} & =(1-R) \ln (1+f)=\frac{\partial B}{\partial b} \ln (1+f),
\end{aligned}
$$


222

$$
\begin{aligned}
\frac{\partial^{2} L L}{\partial b^{2}}= & -\frac{(1-R)^{2}}{B^{2}}-\bar{A}^{K} N^{B K} K^{2}(1-R)^{2}(\ln (N))^{2} \\
\frac{\partial^{2} L L}{\partial b \partial c}= & \frac{\partial^{2} L L}{\partial b^{2}} \ln (1+f) \\
\frac{\partial^{2} L L}{\partial b \partial \gamma}= & -\bar{A}^{K-1} N^{B K} K^{2}(1-R) 0.014 \exp \{\gamma f\} f \ln (d) \ln (N)\left(\bar{A}+S_{m 0}\right), \\
\frac{\partial^{2} L L}{\partial c^{2}}= & \frac{\partial^{2} L L}{\partial b^{2}}[\ln (1+f)]^{2} \\
\frac{\partial^{2} L L}{\partial c \partial \gamma}= & \frac{\partial^{2} L L}{\partial b \partial \gamma} \ln (1+f), \\
\frac{\partial^{2} L L}{\partial \gamma^{2}}= & -\frac{K 0.014 \exp \{\gamma f\} f^{2} \ln (d)\left(\bar{A}+S_{m 0}\right)}{\bar{A}^{2}}\left\{-\bar{A}+0.014 \exp \{\gamma f\} \ln (d) S_{m 0} N^{2 B K}\right. \\
& \left.+\bar{A}^{K} N^{B K}\left[\bar{A}-0.014 \exp \{\gamma f\} \ln (d) S_{m 0}+\left(\bar{A}+S_{m 0}\right) 0.014 \exp \{\gamma f\} \ln (d) K\right]\right\}
\end{aligned}
$$

Defining

$$
H(f)=\frac{0.42278-K \ln (\bar{A})}{b+c \ln (1+f)}, \quad M(f)=\frac{K 0.014 \exp \{\gamma f\} f \ln (d)\left(\bar{A}+S_{m 0}\right)}{\bar{A}}
$$

and $X(f)=\log (1+f)$ as before, the elements of the FIM are the following expected values

$$
\begin{aligned}
I_{11}(b, c, \gamma ; f) & =E\left[-\frac{\partial^{2} L L}{\partial b^{2}}\right]=\frac{\left(\pi^{2} / 6\right)}{[b+c \ln (1+f)]^{2}}+H(f)^{2} \equiv I_{11}(f), \\
I_{12}(b, c, \gamma ; f) & =E\left[-\frac{\partial^{2} L L}{\partial b \partial c}\right]=I_{11}(f) X(f) \equiv I_{12}(f), \\
I_{13}(b, c, \gamma ; f) & =E\left[-\frac{\partial^{2} L L}{\partial b \partial \gamma}\right]=H(f) M(f) \equiv I_{13}(f), \\
I_{22}(b, c, \gamma ; f) & =E\left[-\frac{\partial^{2} L L}{\partial c^{2}}\right]=I_{11}(f)[X(f)]^{2} \equiv I_{22}(f), \\
I_{23}(b, c, \gamma ; f) & =E\left[-\frac{\partial^{2} L L}{\partial c \partial \gamma}\right]=I_{13}(f) X(f) \equiv I_{23}(f), \\
I_{33}(b, c, \gamma ; f) & =E\left[-\frac{\partial^{2} L L}{\partial \gamma^{2}}\right]=[M(f)]^{2} \frac{\left(\bar{A}+S_{m 0}\right) K-S_{m 0}+2 S_{m 0} \bar{A}^{-2 K}}{\left(\bar{A}+S_{m 0}\right) K} \\
& \equiv I_{33}(f) .
\end{aligned}
$$


Again $E$ stands for the mathematical expectation; that is the integral of the quantity multiplied by the density function, or likewise the likelihood function. Typical integration methods were used to find these expressions. Consequently, the FIM at a particular value of $f$ can be expressed as

$$
I(f)=\left(\begin{array}{lll}
I_{11}(f) & I_{11}(f) X(f) & I_{13}(f) \\
I_{11}(f) X(f) & I_{11}(f) X(f)^{2} & I_{13}(f) X(f) \\
I_{13}(f) & I_{13}(f) X(f) & I_{33}(f)
\end{array}\right) .
$$

For an approximate design with $k$ points in its support,

$$
\xi=\left\{\begin{array}{llll}
f_{1} & f_{2} & \cdots & f_{k} \\
p_{1} & p_{2} & \cdots & p_{k}
\end{array}\right\}
$$

the FIM is

$$
M(b, c, \gamma ; \xi)=\left(\begin{array}{lll}
\sum_{i=1}^{k} I_{11}\left(f_{i}\right) p_{i} & \sum_{i=1}^{k} I_{11}\left(f_{i}\right) X\left(f_{i}\right) p_{i} & \sum_{i=1}^{k} I_{13}\left(f_{i}\right) p_{i} \\
\sum_{i=1}^{k} I_{11}\left(f_{i}\right) X\left(f_{i}\right) p_{i} & \sum_{i=1}^{k} I_{11}\left(f_{i}\right) X\left(f_{i}\right)^{2} p_{i} & \sum_{i=1}^{k} I_{13}\left(f_{i}\right) X\left(f_{i}\right) p_{i} \\
\sum_{i=1}^{k} I_{13}\left(f_{i}\right) p_{i} & \sum_{i=1}^{k} I_{13}\left(f_{i}\right) X\left(f_{i}\right) p_{i} & \sum_{i=1}^{k} I_{33}\left(f_{i}\right) p_{i}
\end{array}\right)
$$

and its determinant

$$
\begin{aligned}
\operatorname{det}(M)= & \sum_{i=1}^{k} I_{33}\left(f_{i}\right) p_{i} \sum_{i=1}^{k} I_{11}\left(f_{i}\right) p_{i} \sum_{j>i}^{k} I_{11}\left(f_{j}\right) p_{j}\left[X\left(f_{j}\right)-X\left(f_{i}\right)\right]^{2} \\
& -\sum_{i=1}^{k} I_{11}\left(f_{i}\right) p_{i}\left\{\sum_{j \neq i}^{k} I_{13}\left(f_{j}\right) p_{j}\left[X\left(f_{j}\right)-X\left(f_{i}\right)\right]\right\}^{2} .
\end{aligned}
$$

As mentioned before, we concentrate on designing for loading frequency. In order to compare with the experimental design presented in [1], the same values for $\sigma_{\max }$ and $R$ are adopted as minimal values in our design. The nominal values assumed for the fatigue tests are listed in Table 2.

After some computations, the designs maximising the determinants of the FIMs for C2, CF1 and CF2 when $f \in(0,10]$ are as follows

$$
\xi_{C 2}^{*}=\left\{\begin{array}{cc}
0.1087 & 10 \\
1 / 3 & 2 / 3
\end{array}\right\}
$$


Table 2: Summary of the model parameters for the materials considered and fatigue test conditions in [1] and [19].

\begin{tabular}{lccccccccc}
\hline Mat. & $b$ & $c$ & $\gamma$ & $\begin{array}{c}\lambda \\
{[\mathrm{MPa}]}\end{array}$ & $K$ & $\begin{array}{c}\sigma_{\min _{0}} \\
{[\mathrm{MPa} / \mathrm{s}]}\end{array}$ & $\begin{array}{c}\dot{\sigma}_{0} \\
{[\mathrm{MPa}]}\end{array}$ & $\begin{array}{c}\sigma_{\max } \\
{[\mathrm{MPa}]}\end{array}$ & $R$ \\
\hline $\mathrm{C} 2$ & 0.061 & 0.0105 & 0.24 & 76.1 & 19.8 & 3.1 & 0.2 & 66.4 & 0.3 \\
$\mathrm{CF} 1$ & 0.049 & 0.0066 & 0 & 76.1 & 31.0 & 12.0 & 0.2 & 75.6 & 0.3 \\
$\mathrm{CF} 2$ & 0.052 & 0.0035 & 0.086 & 70.2 & 15.8 & 4.8 & 0.2 & 62.9 & 0.3 \\
\hline
\end{tabular}

$$
\xi_{C F 1}^{*}=\left\{\begin{array}{cc}
0.1229 & 10 \\
1 / 3 & 2 / 3
\end{array}\right\}
$$

$$
\xi_{C F 2}^{*}=\left\{\begin{array}{cc}
0.1181 & 10 \\
1 / 3 & 2 / 3
\end{array}\right\}
$$

The sensitivity function is used to check whether these designs are actually D-optimal or not. Figure 3 shows that this function attains the maximum at the two points of the designs for the three concretes, C2, CF1 and CF2. As mentioned above, since the model is nonlinear, the optimal design will depend on the nominal values of the parameters. A sensitivity analysis versus an alternative choice of the parameters is now performed. For this, different possible true values of the parameters, $\theta_{t}=\left(b_{t}, c_{t}, \gamma_{t}\right)$, around the nominal values will be checked. As a first step, the optimal design for those possible true values is computed, say $\xi_{t}^{*}$. Then the efficiency of the designs (18), (19) and (20), obtained for the nominal values, will be computed using the FIM built with $\theta_{t}$; namely

$$
E f f_{\theta_{t}}\left(\xi^{*}\right)=\left[\frac{\operatorname{det}\left(M\left(\theta_{t}, \xi^{*}\right)\right)}{\operatorname{det}\left(M\left(\theta_{t}, \xi_{t}^{*}\right)\right)}\right]^{\frac{1}{3}} .
$$

This efficiency is computed for values of $b_{t}$ and $c_{t}$ between 0.5 and 1.5 times the nominal values and $\gamma_{t}$ from 0.75 to 1.25 times the nominal value. In all cases the efficiency is over $99 \%$. Since the term $\log (\bar{A})$ appears in the FIM, 


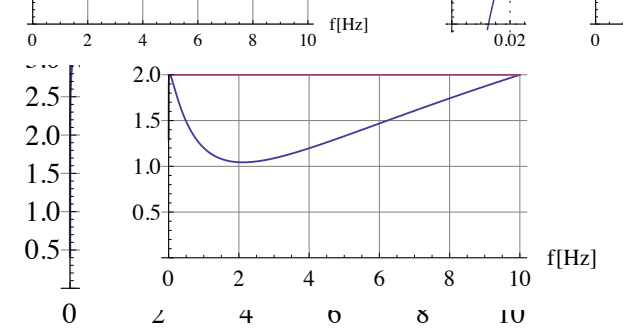

Sensitivity

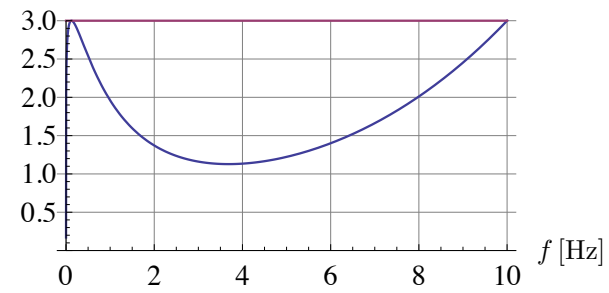

Sensitivity

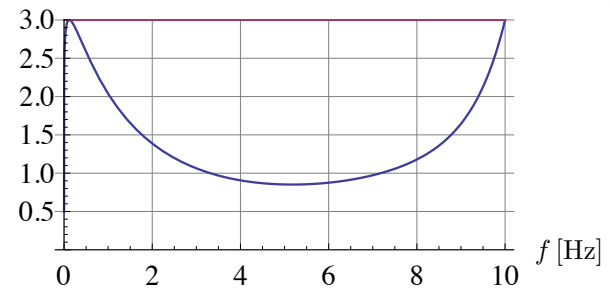

(a)

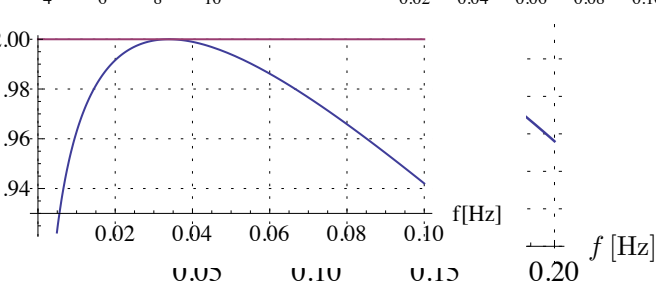

(b)

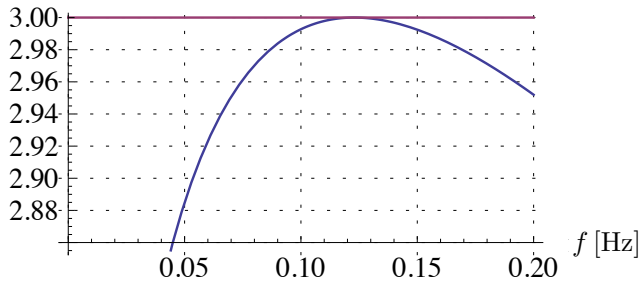

(c)

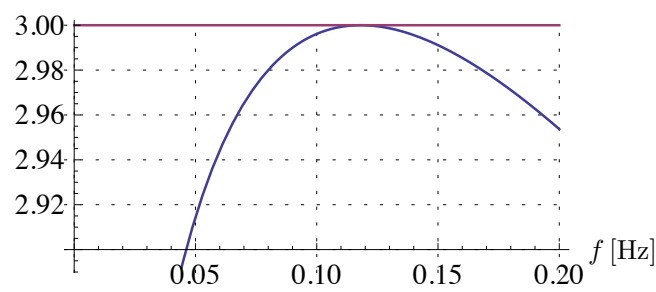

Figure 3: (Complete fatigue model) Sensitivity function for the D-optimal design over the entire frequency range from 0 to $10 \mathrm{~Hz}$ (left column) and the zoomed view at the top-left corner (right column) for (a) $\mathrm{C} 2$, (b) $\mathrm{CF} 1$, and (c) CF2, where the maximum is located at $0.1087 \mathrm{~Hz}, 0.1229 \mathrm{~Hz}$ and $0.1181 \mathrm{~Hz}$, respectively.

the values of $\gamma_{t}$ are chosen to preserve the positiveness of $\bar{A}$. This is the reason why less variation is considered for the parameter $\gamma_{t}$.

The experimental design used in [1] to estimate the parameters was

$$
\xi_{e}=\left\{\begin{array}{cccc}
4^{-2} & 4^{-1} & 4^{0} & 4^{1} \\
1 / 4 & 1 / 4 & 1 / 4 & 1 / 4
\end{array}\right\} .
$$

and the efficiency of this design for the different concretes is $\operatorname{Eff}\left(\xi_{e}, \xi_{C 2}^{*}\right)=$ $0.64, \operatorname{Eff}\left(\xi_{e}, \xi_{C F 1}^{*}\right)=0.6465$ and $\operatorname{Eff}\left(\xi_{e}, \xi_{C F 2}^{*}\right)=0.65$. This means that using the D-optimal design, 35-36\% of experiments can be saved while still obtaining the same statistical results in terms of power of hypotheses tests on the parameters. 


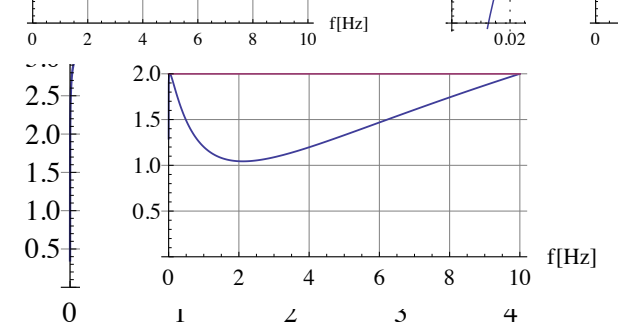

Sensitivity

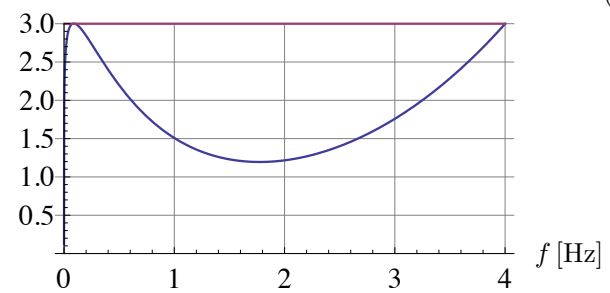

Sensitivity

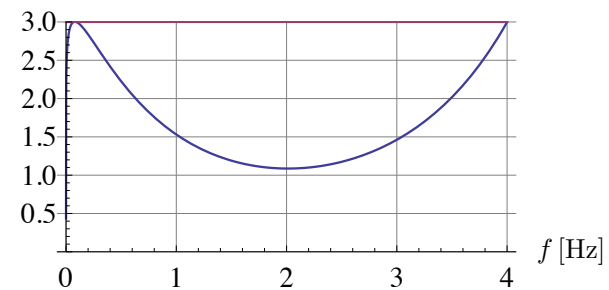

(a)

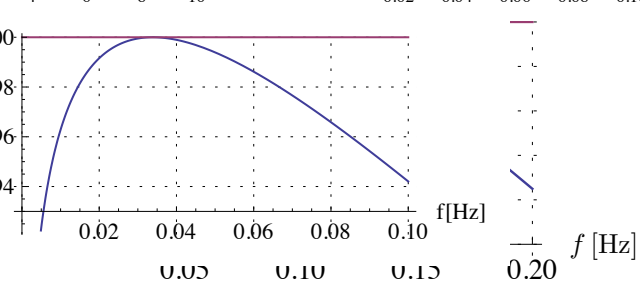

(b)

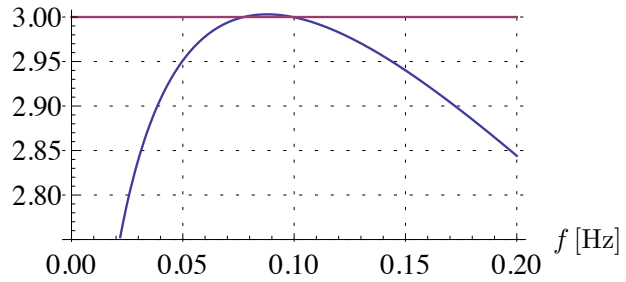

(c)

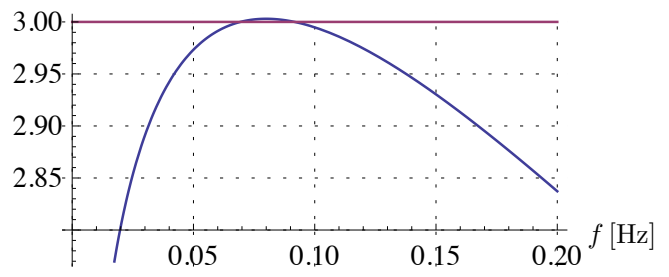

Figure 4: (Complete fatigue model) Sensitivity function for the D-optimal design over the entire frequency range from 0 to $4 \mathrm{~Hz}$ (left column) and the zoomed view (right column) for (a) C2, (b) CF1, and (c) CF2, where the maximum is located at $0.0760 \mathrm{~Hz}, 0.0879 \mathrm{~Hz}$ and $0.0799 \mathrm{~Hz}$, respectively.

249

250
In the following design, we fix $\sigma_{\max }$ and $R$ as given in Table 2 . The optimisation procedure is carried out for the loading frequency $f$ solely in the range of $[0,4] \mathrm{Hz}$. The following results (see Fig. 4) are obtained

$$
\xi_{C 2}^{*}=\left\{\begin{array}{cc}
0.0760 & 4 \\
1 / 3 & 2 / 3
\end{array}\right\}
$$

$$
\xi_{C F 1}^{*}=\left\{\begin{array}{cc}
0.0879 & 4 \\
1 / 3 & 2 / 3
\end{array}\right\}
$$

$$
\xi_{C F 2}^{*}=\left\{\begin{array}{cc}
0.0799 & 4 \\
1 / 3 & 2 / 3
\end{array}\right\}
$$




$$
\xi=\left\{\begin{array}{cccc}
\left(4^{-2}, \sigma_{\max }, 0.3\right) & \left(4^{-1}, \sigma_{\max }, 0.3\right) & \left(4^{0}, \sigma_{\max }, 0.3\right) & \left(4^{1}, \sigma_{\max }, 0.3\right) \\
1 / 4 & 1 / 4 & 1 / 4 & 1 / 4
\end{array}\right\}
$$

Consequently, the efficiencies of the experimental design carried out in [1]

are $64.01 \%, 65.27 \%$ and $64.82 \%$ for $\mathrm{C} 2, \mathrm{CF} 1$ and $\mathrm{CF} 2$, respectively.

\section{Summary and conclusions}

We have developed a general procedure for obtaining the optimal design in a fatigue characterisation process and applied the concept of optimal experimental design to a fatigue model based on the initial Weibull distribution of the static strength of concrete-like materials. The Fisher Information Matrix (FIM) is obtained both for the simplified and complete fatigue model. In particular, the optimisation process is carried out for given maximum stress, stress ratio and loading frequency ranges and a D-optimal design is obtained. The previously carried out experimental designs is shown to be $87 \%$ and $65 \%$ efficient for the simplified and complete fatigue model, respectively. In other words, $13 \%$ or $35 \%$ of the experiments could have been saved if the attained optimal experimental designs had been considered. In addition, a robustness analysis was carried out to check the dependence of the choice of the nominal model parameters; the efficiency surpasses $99 \%$ when $b$ and $c$ vary between $50 \%$ and $150 \%$, and $\gamma$ changes between $75 \%$ and $125 \%$ of their respective nominal values.

Even though the procedure has been demonstrated for a chosen fatigue model, it is applicable to any fatigue model for any other material.

\section{Acknowledgements}

The authors acknowledge the financial support from the Ministerio de Economía y Competitividad and funds FEDER BIA2015-68678-C2-1-R, MTM201347879-C2-1-P, MTM2013-47879-C2-2-P, Junta de Castilla-La Mancha (PEII2014-016-P) and Junta de Castilla y León (SA130U14). 


\begin{tabular}{|c|c|c|}
\hline & $\sigma_{\max }$ & Maximum stress under fatigue loading \\
\hline & $\sigma_{\min }$ & Minimum stress under fatigue loading \\
\hline & $\sigma_{\max _{0}}$ & Equivalent static value of the dynamic stress, $\sigma_{\max }$ \\
\hline & $\triangle \sigma$ & Stress range $\sigma_{\max }-\sigma_{\min }$ \\
\hline & $R$ & Stress ratio defined as $\sigma_{\min } / \sigma_{\max }$ \\
\hline & $\sigma_{f}$ & Failure stress \\
\hline & $\sigma_{\min _{0}}$ & Fatigue endurance limit \\
\hline & $\dot{\sigma}_{0}$ & Loading rate of the quasi-static test \\
\hline & $\sigma_{c}$ & Critical stress, can be compressive $\left(f_{c}\right)$, tensile $\left(f_{t}\right)$ \\
\hline & $N$ & Number of cycles to failure (fatigue life) \\
\hline & $\lambda, K$ & Scale and shape parameter of the Weibull distribution \\
\hline & $S_{m x}$ & Normalised maximum stress $\sigma_{\max } / \lambda$ \\
\hline & $S_{m 0}$ & Normalised minimum stress $\sigma_{\min _{0}} / \lambda$ \\
\hline 77 & $P F$ & Probability of failure in any point of the domain $\sigma_{f}-\ln N$ \\
\hline & $f$ & Loading frequency employed in a fatigue test \\
\hline & $b, c$ & Parameter that adjusts the relation between $\ln N$ and $f, R$ \\
\hline & $\gamma$ & $\begin{array}{l}\text { Coefficient that takes into consideration of loading frequency } \\
\text { for material strength under dynamic loading }\end{array}$ \\
\hline & $\xi$ & approximate design as a probability measure \\
\hline & $x$ & the vector of covariates defining a particular experiment \\
\hline & $\beta$ & vector of model parameters \\
\hline & $E[\cdot]$ & mathematical expectation \\
\hline & $L L(\beta ; t, x)$ & the log-likelihood function of the observation $t$ at $x$ \\
\hline & $I(\beta ; x)$ & Fisher Information Matrix at point $x$ \\
\hline & $M(\beta ; \xi)$ & Fisher Information Matrix at design $\xi$ \\
\hline
\end{tabular}

\section{Appendix}

\section{References}

[1] L. Saucedo, R. C. Yu, A. Medeiros, X. X. Zhang, and G. Ruiz. A probabilistic fatigue model based on the initial distribution to consider frequency effect in plain and fiber reinforced concrete. International Journal of Fatigue, 48:308-318, 2013.

[2] O. Graf and E. Brenner. Experiments for investigating the resistance of concrete under often repeated compression loads (in German). Bulletin No. 76 and 83, Deutscher Ausschuss fr Eisenbeton, 1934. 
[3] C. E. Kesler. Effect of speed of testing on flexural fatigue strength of plain concrete. Proceedings of Highway Research Board, 32:251-258, 1953.

[4] G. M. Nordby. Fatigue of concrete - A review of research. ACI Journal, pages 191-220, 1958.

[5] H. Rusch. Researches toward a general flexural theory for structural concrete. ACI Journal, 57(1):1-28, 1960.

[6] J. W. Mudock. A critical review of research on fatigue of plain concrete. Bulletin No. 475, Engineering Experiment Station, University of Illinois-Urbana, pages 1-15, 1965.

[7] K. Aas-Jakobsen. Fatigue of concrete beams and columns. Bulletin No. 70-1, NTH Institutt for Betongkonstruksjoner, Trondheim, 1970.

[8] M.E. Awad and H. K. Hilsdorf. Strength and deformation characteristics of plain concrete subjected to high repeated and sustained loads. Civil Engineering Studies, Structural Research Series No. 372, 266 pp., Department of Civil Engineering, University of Illinois-Urbana, Feb. 1971.

[9] P. R. Sparks and J. B. Menzies. The effect of rate of loading upon the static and fatigue strengths of plain concrete in compression. Magazine of Concrete Research, 25(83):73-80, Sept. 1973.

[10] ACI Committee 215. Considerations for design of concrete structures subjected to fatigue loading. ACI Journal, pages 97-121, 1974.

[11] R. Tepfers and T. Kutti. Fatigue strength of plain, ordinary and lightweight concrete. ACI Journal, 76(5):635-652, 1979.

[12] R. Tepfers. Tensile fatigue strength of plain concrete. ACI Journal, 76(8):919-933, 1979.

[13] T. T. C. Hsu. Fatigue of plain concrete. ACI Journal, 78:292-304, 1981.

[14] K. Furtak. Ein verfahren zur berechnung der betonfestigkeit unter schwellenden belastungen. Cement and Concrete Research, 14(6):855-865, 1984. 
[15] B. H. Oh. Fatigue-life distributions of concrete for various stress levels. ACI Materials Journal, 88(2):122-128, 1991.

[16] B. Zhang, D. V. Philips, and K. Wu. Effect of loading frequency and stress reversal of fatigue life of plain concrete. Magazine of Concrete Research, 48(177):361-375, Dec. 1996.

[17] H. Li, M. H. Zhang, and J. P. Ou. Flexural fatigue performance of concrete containing nano-particles for pavement. International Journal of Fatigue, 29:1291-1301, 2007.

[18] D. F. Zhao, Q. Y. Chang, J. H. Yang, and Y. P. Song. A new model for fatigue life distribution of concrete. Key Engineering Materials, 348-349:201-204, 2007.

[19] A. Medeiros, X. X. Zhang, G. Ruiz, R. C. Yu, and M. S. L. Velasco. Effect of the loading frequency effect on the compressive fatigue behavior of plain and fiber-reinforced concretes. International Journal of Fatigue, 70:342-350, 2015.

[20] O. Plekhov, M. Paggi, O. Naimark, and A. Carpinteri. A dimensional analysis interpretation to grain size and loading frequency dependencies of the Paris and Wöhler curves. International Journal of Fatigue, 33(3):477-483, 2011.

[21] G. Ruiz, A. Medeiros, and X. X. Zhang. Experimental study of loading frequency effect on compressive fatigue behavior of plain and fiber reinforced concretes. Anales de Mecánica de la Fractura, 29:535-540, 2011.

[22] D. R. Cox. Regression Models and Life-Tables. Journal of the Royal Statistical Society, Series B, 34(2):187-220, 1972.

[23] J. López-Fidalgo and M. J. Rivas-López. Optimal experimental designs for partial likelihood information. Computational Statistics \& Data Analysis, 71:859-867, 2014.

[24] M. J. Rivas-López, J. López-Fidalgo, and R. del Campo. Optimal experimental designs for accelerated failure time with Type I and random censoring. Biometrical Journal, 56(5):819-837, 2014. 
[25] S. D. Silvey. Optimal Design. Chapman \& Hall, London, 1980.

[26] J. Kiefer. General Equivalence Theory for Optimum Designs (Approximate Theory). The Annals of Statistics, 2(5):849-879, 1974.

[27] A. Pázman. Foundations of Optimum Experimental Design. D. Reidel publishing company, Dordrecht, 1986.

[28] CEB-FIP. Constitutive modeling of high strength/high performance concrete. FIB Bulletin, 42, 2008.

[29] J. C. Lagarias. Eurler's constant: Euler's work and modern developments. Bulletin of the American Mathematical Society, 50(4):527-628, Oct. 2013.

[30] M. Imhof, J. López-Fidalgo and W.K. Wong. Efficiences of Optimal Aproximate Designs for Small Samples. Statistica Neerlandica, 55:301-315, 2001.

[31] J. López-Fidalgo, Ch. Tommasi and C. Trandafir. An optimal experimental design criterion for discriminating between non-Normal models. Journal of the Royal Statistical Society, Series B, 69(2):231-242, 2007. 